\title{
Analysis of Long Time Series of Polar Motion
}

\author{
H. Schuh, B. Richter and S. Nagel \\ Deutsches Geodätisches Forschungsinstitut (DGFI) Marstallplatz 8, \\ 80539 München, Germany e-mail: schuh@dgfi.badw.de
}

\begin{abstract}
Two long time series of polar motion were analysed with respect to a linear drift, decadal variations, Chandler wobble and annual wobble: the $\mathrm{C} 01$ series published by the International Earth Rotation Service (IERS) and the pole series which J. Vondrák, obtained by reanalysis of the classical astronomical observations using the HIPPARCOS reference frame (1899.7-1992.0). By a least-squares fit the linear drift of the pole, usually called 'secular polar motion,' was determined to 3.31 milliarcseconds/year (mas/yr) toward $76.1^{\circ}$ West longitude. For this fit the a priori correlations within each pair of pole coordinates were taken into account, and the weighting function was calculated by estimation of empirical variance components. The decadal variations of the pole path were determined by Fourier analysis. Using a sliding window analysis, the variability of the periods, the amplitudes and the phases of the Chandler wobble and annual wobble was investigated. The variances of the results and the number of iterations needed to get a convergence in the nonlinear approach show that the new time series by Vondrák is more homogeneous and consistent than the IERS C01 series.
\end{abstract}

\section{Introduction}

In polar motion research there are - more than 100 years after the discovery of polar motion by Küstner (1888) and the detection of a characteristic non-annual free motion in the observed polar motion by Chandler (1891) - still a lot of questions waiting for a solution. The main open points are:

- Is there a long-term drift of the pole with respect to the Earth's surface?

- If so, what are the main causes for this drift which is usually called 'polar wandering' or 'secular polar motion?'

- What are the causes of the decadal variations of polar motion which can be seen in the observations?

- Why is the amplitude of the Chandler wobble (CW) not steadily decreasing with time due to damping, and what are the excitation mechanisms of the CW? 
- What are the reasons for the rapid phase jumps and/or the apparent strong variations of the $\mathrm{CW}$, which have been reported by many scientists from analysis of polar motion time series?

The reasons for the investigation presented in our paper are twofold:

- New precise and consistent time series of polar motion exist which will be described in the next paragraph.

- Powerful computers are available which allow us to invert big matrices within a short time. Thus, the stochastical model of the analyses can be extended and completed as will be shown in the paper.

Starting from these prerequisites the different components of polar motion and their time variability were investigated. The goal of this study is to fully describe what can be seen in the data. The results are supposed to be the subject of further interpretations.

\section{Long Time Series of Polar Motion}

Several time series of polar motion were analysed with respect to a linear drift. The linear model was then combined with periodical models of the Chandler wobble (CW) and the annual wobble (AW). The decadal variations were investigated by Fourier analyses. Finally, the CW and the AW parameters were repeatedly determined by a sliding window analysis.

The following long time series of polar motion were analysed:

1a) The C01 series (1861.0-1997.0) published by the International Earth Rotation Service (IERS); it will be called IERS C01 (1861.0-1997.0) in the paper.

1b) Regular astronomical observations by the International Latitude Service (ILS) started in 1899 and because we wanted to compare the C01 series with the data series described later on, we also looked at a second series which is a subset of the first one for the time interval from 1899.7 till 1992.0. This series will be called IERS C01 (1899.7-1992.0).

2a) The pole series OA97 (1899.7-1992.0) obtained by re-analysis of optical astrometry observations referred to the HIPPARCOS catalogue as described by Vondrák (1999). This series based on 4.3 million observations was used for the determination of the linear drift and for the sliding window analyses described in this paper.

2b) We also used a new time series recently received from Vondrák (Vondrák, 2000 , this issue) in order to compare the results for the linear drift in polar motion. This new series, which will be called OA99 (1899.7-1992.0) in the paper is based on 4.5 million observations. This is more than the OA97 series although the observations taken at Ukiah since 1960 were neglected because of probable local or regional movements of that point. The OA99 series also refers to a slightly different reference frame due to proper motion corrections. 


\section{Secular polar motion and decadal variations}

\subsection{Model}

The model used contains a purely linear part for 'secular polar motion' (offset and drift parameters $a, b, c, d)$ and two periodical elliptical motions representing the Chandler wobble (parameters $R_{1 a}, R_{1 b}, \omega_{1}, \phi_{1 a}, \phi_{1 b}$ ) and the annual wobble (parameters $\left.R_{2 a}, R_{2 b}, \omega_{2}, \phi_{2 a}, \phi_{2 b}\right)$ :

$$
\begin{aligned}
& x_{p}=a \cdot t+b+R_{1 a} \cos \left(\phi_{1 a}+\omega_{1} \cdot t\right)+R_{2 a} \cos \left(\phi_{2 a}+\omega_{2} t\right) \\
& y_{p}=c \cdot t+d+R_{1 b} \sin \left(\phi_{1 b}+\omega_{1} \cdot t\right)+R_{2 b} \sin \left(\phi_{2 b}+\omega_{2} t\right)
\end{aligned}
$$

All parameters were estimated simultaneously by a least-squares fit of which details will be given in the next two paragraphs.

\subsection{Choice of the weighting function}

One important issue in least-squares parameter estimation is the right choice of the weight matrix $P$, i.e. the determination of an appropriate weighting function. In our study the weights $p_{i}$ were chosen according to three different weighting functions:

1.) $p_{i}=1$,

means equal weights, i.e. each data point contributes equally to the final results. This simple method was used in most of the analyses reported in the literature (see the comprehensive tables given in McCarthy and Luzum (1996), Korsun and Yatskiv (1999) and Gross and Vondrák (1999)). This approach does however not take into account that the precision of the measurements has considerably increased during the century.

2.) $p_{i}=$ const $/ \sigma_{i}^{2}$,

with $\sigma_{i}^{2}$ being the variances of the 'observed' pole coordinates as given in the time series. When using this weighting function which is usually recommended in the textbooks on least-squares analysis, care has to be taken that a small number of observations does not influence and perhaps bias the results whereas the other observations - although formally entered into the least-squares fit are almost neglected. E.g. a difference by a factor 10 of the formal errors $\sigma_{i}$ of the observations will cause differences of the weights by a factor 100 .

3.) $p_{i}=$ const $/\left(\sigma_{i}^{2}+\sigma_{0}^{2}\right)$,

with $\sigma_{0}^{2}$ being a constant summand. This approach allows us to take into account the different precisions of the data but not as much as the formal errors indicate. A reasonable value for $\sigma_{0}^{2}$ can be obtained by a $\chi^{2}$-test when comparing the ( $a$ posteriori) residuals with the a priori errors of the observables. This procedure is called estimation of empirical variance components. Fig. 1 shows the three weighting functions as described above depending on the $a$ priori formal errors $\sigma_{i}$. The constant $\sigma_{0}^{2}$ was set to $0.054 \operatorname{arcsec}^{2}$.

\subsection{A priori correlations within each pair of pole coordinates}

In both time series OA97 and OA99 calculated by Vondrák $(1999,2000)$ also correlation coefficients are given within each pair of pole coordinates $x_{p}, y_{p}$. They are usually between 0.2 and 0.5 , some of them reach 0.9 and more. Therefore, 


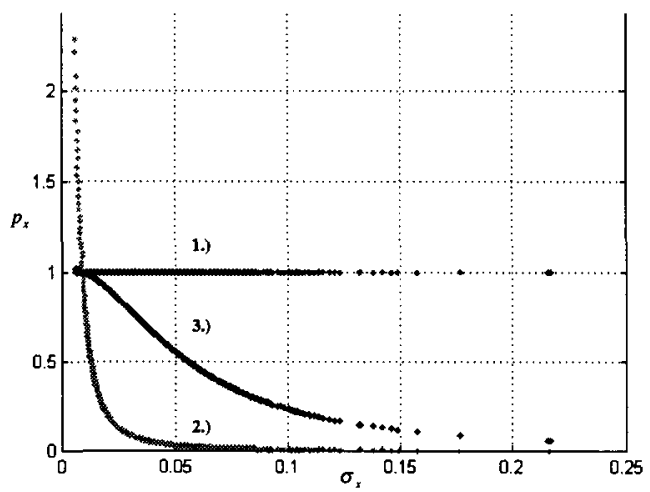

Figure 1. Three weighting functions described in this paragraph depending on the it a priori formal errors $\sigma_{i}$. The constant $\sigma_{0}^{2}$ was set to $0.054 \operatorname{arcsec}^{2}$.

two solutions were carried out, firstly the 'uncorrelated' (standard) solution neglecting the correlations and secondly a solution in which the given correlations within the observables were considered in the least-squares fit ('correlated approach'). In the latter approach the full variance-covariance matrix has to be calculated. Although the inversion of this very big matrix requires relatively powerful computers, it is not a real problem, today.

\subsection{Determination of the linear drift of polar motion}

The results of the various least-squares fits are summarized in Table 1. It can be seen that the choice of appropriate weights is essential; both extreme cases $\left(p_{i}=1, p_{i}=\right.$ const $\left./ \sigma_{i}^{2}\right)$ seem not to be reasonable. In particular, with approach 2.) the results are biased very much towards the more precise observations from 1960 till 1980 although these data cover only a relatively short time span.

The correlated approach with a weighting function determined by the empirical variance component estimation method can be considered as the best one. Thus, we obtained from the pole series OA97 (1899.7-1992.0) as the most plausible result for the linear drift of the pole in the 20th century a motion of 3.31 $\mathrm{mas} / \mathrm{yr}$ in the direction of $76.1^{\circ}$ West longitude. This is considerably smaller than the drifts obtained from the two IERS C01 series, which are 4.4 mas/yr in the direction of $78.1^{\circ}$ West longitude for the series C01 (1899.0-1992.0) and $4.0 \mathrm{mas} / \mathrm{yr}$ in the direction of $77.4^{\circ}$ West longitude for the series $\mathrm{C} 01$ (1861.01997.0). The linear drift from the second re-analysis series OA99 (1899.7-1992.0) is $2.81 \mathrm{mas} / \mathrm{yr}$ in $75.4^{\circ}$ West longitude (correlated approach, weighting function 3.)) and thus even smaller than from the OA97 series. The linear polar motion is most probably caused by post-glacial rebound as described by Vondrák (1999), where further references to that topic are given.

\subsection{Decadal periods}

Now, the long time series were entered into standard Fourier analysis. For the re-analysis series OA97 (1899.7-1992.0) the peaks corresponding to the main 
Table 1. Linear drift of the pole determined from different data series; those results are marked which are considered to be the most plausible ones

\begin{tabular}{|c|c|c|c|c|c|c|}
\hline & \multicolumn{2}{|c|}{$\begin{array}{l}p_{i}=1.0 \\
\text { sec. p.m. } \\
\text { [mas } / \mathbf{y r}]\end{array}$} & \multicolumn{2}{|c|}{$\begin{array}{l}\quad p_{i}=\frac{\text { const }}{\sigma_{i}^{2}} \\
\text { sec. p.m. } \\
{[\mathrm{mas} / \mathrm{yr}]}\end{array}$} & \multicolumn{2}{|c|}{$\begin{array}{l}\quad p_{i}=\frac{\text { const }}{\sigma_{i}^{2}+\sigma_{0}^{2}} \\
\text { sec. } \text { p.m. } \\
{[\mathrm{mas} / \mathrm{yr}]}\end{array}$} \\
\hline $\begin{array}{c}\text { IERS C01 } \\
(1899-1992)\end{array}$ & $\begin{array}{c}4.38 \\
\pm 0.08\end{array}$ & $\begin{array}{c}77.43 \\
\pm 1.06\end{array}$ & $\begin{array}{c}6.02 \\
\pm 0.13\end{array}$ & $\begin{array}{r}85.16 \\
\pm 1.25\end{array}$ & $\begin{array}{c}4.43 \\
\pm 0.08\end{array}$ & $\begin{array}{l}78.15 \\
\pm 1.00\end{array}$ \\
\hline $\begin{array}{c}\text { IERS C01 } \\
(1861-1997)\end{array}$ & $\begin{array}{c}3.58 \\
\pm 0.05\end{array}$ & $\begin{array}{r}75.53 \\
\pm 0.85\end{array}$ & $\begin{array}{c}4.49 \\
\pm 0.10\end{array}$ & $\begin{array}{c}82.29 \\
\pm 1.24\end{array}$ & $\begin{array}{c}4.00 \\
\pm 0.06\end{array}$ & $\begin{array}{l}77.36 \\
\pm 0.77\end{array}$ \\
\hline $\begin{array}{c}\text { OA97 } \\
(1899-1992) \\
\text { uncorrelated }\end{array}$ & $\begin{array}{c}3.38 \\
\pm 0.05\end{array}$ & $\begin{array}{l}78.69 \\
\pm 0.80\end{array}$ & $\begin{array}{c}3.80 \\
\pm 0.04\end{array}$ & $\begin{array}{c}82.73 \\
\pm 0.65\end{array}$ & $\begin{array}{c}3.40 \\
\pm 0.05\end{array}$ & $\begin{array}{c}79.27 \\
\pm 0.76\end{array}$ \\
\hline $\begin{array}{c}\text { OA97 } \\
(1899-1992) \\
\text { correlated }\end{array}$ & $\begin{array}{c}3.27 \\
\pm 0.05\end{array}$ & $\begin{array}{c}75.11 \\
\pm 0.84\end{array}$ & $\begin{array}{c}3.78 \\
\pm 0.04\end{array}$ & $\begin{array}{c}80.59 \\
\pm 0.65\end{array}$ & $\begin{array}{c}3.31 \\
\pm 0.05\end{array}$ & $\begin{array}{l}7 \overline{6} .08 \\
\pm 0.80\end{array}$ \\
\hline $\begin{array}{c}\text { OA99 } \\
(1899-1992) \\
\text { uncorrelated }\end{array}$ & $\begin{array}{c}2.85 \\
\pm 0.05\end{array}$ & $\begin{array}{c}73.55 \\
\pm 0.93\end{array}$ & $\begin{array}{c}2.49 \\
\pm 0.04\end{array}$ & $\begin{array}{c}71.54 \\
\pm 0.95\end{array}$ & $\begin{array}{c}2.81 \\
\pm 0.04\end{array}$ & $\begin{array}{c}73.46 \\
\pm 0.90\end{array}$ \\
\hline $\begin{array}{c}\text { OA99 } \\
\text { (1899-1992) } \\
\text { correlated }\end{array}$ & $\begin{array}{c}2.81 \\
\pm 0.04\end{array}$ & $\begin{array}{l}73.46 \\
\pm 0.90\end{array}$ & $\begin{array}{c}2.48 \\
\pm 0.04\end{array}$ & $\begin{array}{r}73.04 \\
\pm 0.96\end{array}$ & $\begin{array}{c}2.81 \\
\pm 0.04\end{array}$ & $\begin{array}{l}75.45 \\
\pm 0.90\end{array}$ \\
\hline
\end{tabular}

decadal periods were obtained at prograde $77 \mathrm{yr}, 37 \mathrm{yr}, 26 \mathrm{yr}, 17 \mathrm{yr}, 9 \mathrm{yr}$ and at retrograde $86 \mathrm{yr}, 28 \mathrm{yr}, 19 \mathrm{yr}, 15 \mathrm{yr}, 7 \mathrm{yr}$ (Fig. 2). For most of these highly elliptic motions the prograde components are bigger than the corresponding (i.e. with approximately equal periods) retrograde ones. An exception is the so-called Markowitz wobble around 30 years where the retrograde part is dominant. Without showing the detailed results of similar analyses of the $\mathrm{C} 01$ series it should be mentioned that the IERS C01 (1861.0-1997.0) series shows even clearer the long periods around $80 \mathrm{yr}$ and $30 \mathrm{yr}$, in spite of the poor precision of the pole coordinates measured in the 19th century. Causes for decadal variations of polar motion are global mass redistributions in the atmosphere, the hydrosphere and the cryosphere (Jochmann, 1993), e.g. the redistribution of water storage on the Earth (Eubanks, 1993; Jochmann, 1999). Other possible causes are geomagnetic core-mantle coupling (Greiner-Mai, 1993), topographic torques at the core-mantle boundary (Hide, 1995) or inner-core rotation (Greiner-Maj et al., 1999).

\section{Annual Wobble (AW) and Chandler Wobble (CW)}

\subsection{Fourier Analysis}

Concentrating on the AW and CW the Fourier analysis of the re-analysis series OA97 (1899.7-1992.0) reveals three nearby peaks indicating a prograde $\mathrm{CW}$ (429d, 436d, 450d) and a large prograde annual variation (Fig. 3). A small retrograde annual variation can also be seen, i.e. the $\mathrm{AW}$ is a slightly elliptic motion whereas the $\mathrm{CW}$ is a circular motion. The Fourier spectra of both IERS C01 series (not presented here) show very similar periods around the mean CW period as the re-analysis series. However, the (small) retrograde annual variation cannot be seen as clearly as in Figure 3 . 
Fourier spectrum $\left(x_{p}-i^{*} y_{p}\right)$ series OA97 (1899.7-1992.0)

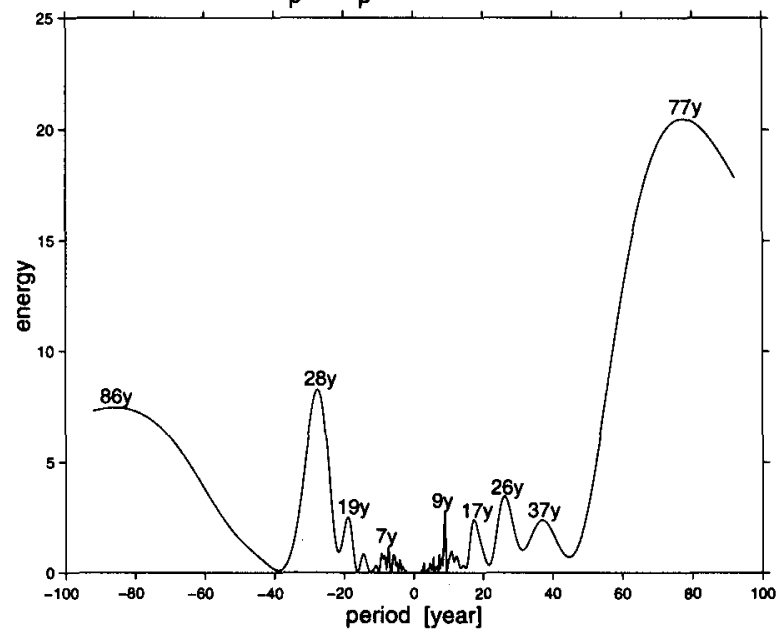

Figure 2. Fourier spectrum of OA97 (1899.7-1992.0) for the decadal periods.

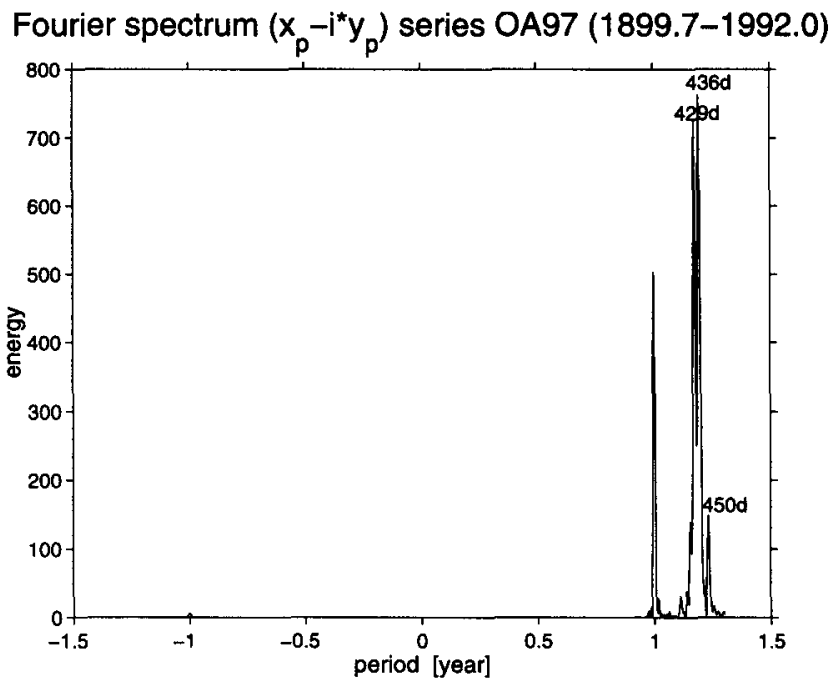

Figure 3. Fourier spectrum of OA97 (1899.7-1992.0) for periods shorter than 1.5 years. 


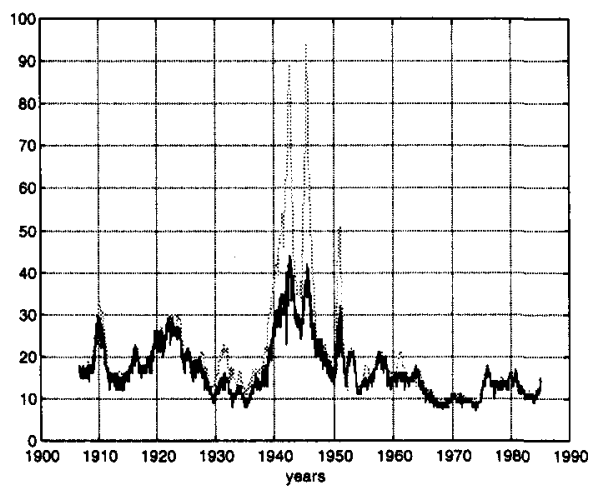

Figure 4. Number of iterations needed to get a sufficient convergence in each of the windows of the sliding analysis.

period as the re-analysis series. However, the (small) retrograde annual variation cannot be seen as clearly as in Figure 3 .

\subsection{Sliding window analysis of the $\mathrm{CW}$ and $\mathrm{AW}$ parameters}

The variations of the Chandler wobble (CW) and of the annual wobble (AW) were investigated by a sliding window analaysis. The window size was set to 13.76 years which corresponds to twice the beat of the AW and a mean CW. In each window the $\mathrm{CW}$ and $\mathrm{AW}$ parameters were determined by a least-squares fit. As the model (eq. 1,2) is nonlinear with respect to the frequencies $\omega_{1}, \omega_{2}$ and phases $\phi_{1 a}, \phi_{1 b}, \phi_{2 a}, \phi_{2 b}$ of the $\mathrm{CW}$ and of the $\mathrm{AW}$, the equations had to be linearized first. Then, starting from good a priori values, the solutions were obtained by iterations. As the quality of the pole data is usually the higher the later they were observed, the window was not moved from the beginning to the end of the time series but in backward direction, i.e. starting with the more precise recent observations and then moving back to the past in very short time steps. When a sufficient convergence was obtained, the iterations were stopped. Although in most of the $13.76 \mathrm{yr}$ time windows the solution converged rather quickly, small differences between the IERS C01 (1899.7-1992.0) and the OA97 (1899.7-1992.0) series can be seen when setting the convergence criterion rather high (i.e. where to stop the iterations). Figure 4 shows the number of iterations needed in each of the $13.76 \mathrm{yr}$ windows for the two data sets. In many windows, significantly fewer iterations had to be carried out for the OA97 (1899.7-1992.0) series compared to the IERS C01 (1899.7-1992.0) series to achieve a convergence. This is probably due to the higher consistency and homogeneity of the re-analysis series OA97 (1899.7-1992.0).

\subsection{Variations of the Chandler wobble (CW) parameters and of the annual wobble (AW) parameters}

By solving for all(!) parameters (amplitudes, phases and periods) of the CW and of the AW in the least-squares fit of the sliding window analyses, the model is more 'general' than in former similar analyses, e.g. Vondrák (1999). We used 


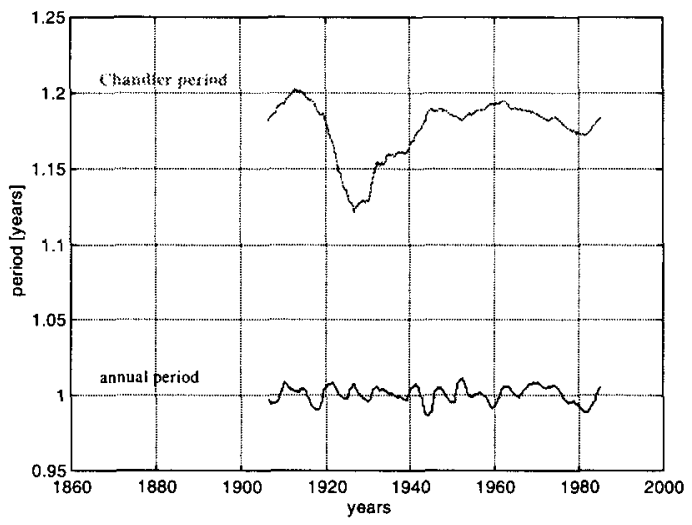

Figure 5. Apparent variation of $\mathrm{CW}$ periods and $\mathrm{AW}$ periods from reanalysis series OA97 (1899.7-1992.0).

the same a priori weights which had been empirically determined by the method described in paragraph 3.2 and entered the $a$ priori correlations (see paragraph 3.3) into the fit. Figures 5, 6 and 7 show the variations of the Chandler periods, amplitudes (semi-major and semi-minor axes) and phases as obtained for the series OA97 (1899.7-1992.0). The parameters of the CW change rapidly, e.g the Chandler amplitude (semi-major and semi-minor axes) varies by a factor of 4 and the Chandler period varies between $1.13 \mathrm{yr}$ and $1.20 \mathrm{yr}$ ( $413 \mathrm{~d}$ and $439 \mathrm{~d}$ ). During the time of the minimum CW amplitude (around 1930) the CW period was extremely short. Also rapid changes of the phases occurred, e.g. the wellknown 'phase jump' of the CW around 1920 and further ones, e.g. 1930-1933 and 1975-1980. However, it has to be kept in mind that the phases, and hence the detected 'phase jumps,' always depend on the time origin $t_{0}$ to which the time $t$ refers. In our analyses $t_{0}$ was set to 1945 . Thus, the variability of the phases vanishes at 1945 and increases with the time distance from 1945. In spite of that fact and of the dependence between period and phase of any periodic oscillation (similar to offset and drift parameter of a linear model) we think that the results are more reliable than previous ones and justify the effort of programming and the expense of computer capacity and time.

The variability of the CW period is clearly revealed by analysing the data series in relatively short time windows of about 14 years. These results can be interpreted that there is either a second wobble nearby the $\mathrm{CW}$ and what we see is a beat of the two (or more) oscillations or - and this seems to be more likely - an excitation of the CW by a quasi-periodic force acting irregularly on the Earth.

The figures 5, 6 and 7 show also the variations of the periods, amplitudes (semi-major and semi-minor axes) and phases of the annual wobble. We can see that the variability is smaller but faster than that of the CW parameters.

We also submitted the long IERS C01 (1861.0-1997.0) time series to a sliding window analysis. Despite its poor quality in the 19 th century - the series allows us to determine the CW and AW parameters already from 1861 


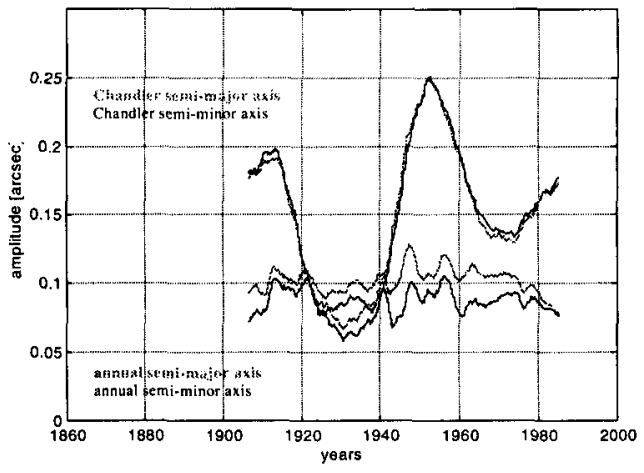

Figure 6. Apparent variation of $\mathrm{CW}$ amplitudes and $\mathrm{AW}$ amplitudes from reanalysis series OA97 (1899.7-1992.0).

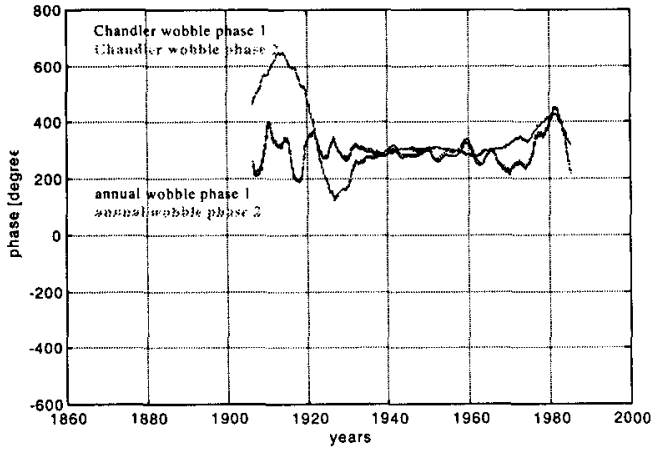

Figure 7. Apparent variation of $\mathrm{CW}$ phases and $\mathrm{AW}$ phases from reanalysis series OA97 (1899.7-1992.0). 
onwards (again moving the window backwards to the past). They vary in the same range as the parameters determined in the 20th century.

\section{Conclusions}

Two long time series of polar motion were analysed with respect to linear drift, decadal variations, Chandler wobble and annual wobble: the C01 series published by the IERS, Paris and the series obtained by re-analysis of the optical observations within the HIPPARCOS frame (Vondrák, 1999, 2000).

The weights used in the least-squares fit were chosen carefully because all results strongly depend on the weighting function. The empirical variance component estimation method was applied to determine an appropriate weighting function. A priori correlations within each pair of pole coordinates (only available for the re-analysis series) were also taken into account in the least-squares fit. As the most reliable result a linear drift of polar motion in the 20th century of $3.31 \mathrm{mas} / \mathrm{yr}$ in the direction of $76.1^{\circ}$ West longitude was obtained from the re-analysis series (OA97) (1899.7-1992.0) using the correlated approach. This linear drift is considerably smaller than the one derived from the IERS C01 series.

From a sliding window analysis it can be seen that the new time series OA97 obtained by re-analysis is more consistent than the IERS C01 series, probably due to the consistent and more comprehensive treatment of the astronomical observations by Vondrák (1999), e.g. by considering plate motions of the observing sites. Both the $\mathrm{CW}$ and - to a less extent - the $\mathrm{AW}$ are rather unstable and seem to be modulated with other nearby periodic variations or excited by irregular driving forces. Obviously, a rapid variability of the $\mathrm{CW}$ parameters can hardly be detected if the time series is analysed in relatively long time windows, as e.g. was done by Wilson and Vicente (1997) with windows of 31 years (1900$1930,1931-1961,1962-1992)$. Taking the mean values for the CW periods and amplitudes in those three time intervals from figure 5 , we see that those averaged results do not differ very much.

\section{References}

Chandler, S.C., 1891, On the variation of latitude, Astron. J., 248, 59-61.

Eubanks, T.M., 1993, Variation in the orientation of the Earth, Contributions of Space Geodesy to Geodynamics: Earth Dynamics, ed. by D.E. Smith and D.L. Turcotte, AGU Geodynamics Series, 24, 1-54.

Greiner-Mai, H., 1993, Decade Variations of the Earth's Rotation and Geomagnetic Core-Mantle Coupling, J. Geomag. Geoelectr., 45, 1333-1345.

Greiner-Mai, H., Jochmann, H., and Barthelmes, F., 1999, Influence of possible inner-core motions on the polar motion and the gravity field, Phys. Earth Planet. Inter., 117, 81-93.

Gross, R.S. and J. Vondrák, 1999, Astrometric and space-geodetic observations of polar wander, Geophys. Res. Lett. 26, 2085-2088.

Jochmann, H., 1993, Earth Rotation and Global Change, Adv. Space Res., 13, 271-280. 
Hide, R., 1995, The topographique torque on a bounding surface of a rotating gravitating fluid and the excitation by core motions of decadal fluctuations in the Earth's rotation, Geophys. Research Letters, 22, 961-964.

Korsun, A.A. and Ya.S. Yatskiv, 1999, Comments on slow latitude variations and secular motion of the Earth's pole, Journées 1998, Syst. de Réf. SpatioTemporels, Paris, ed. by N. Capitaine, Obs. de Paris, UMR 8630/CNRS, $223-227$.

Küstner, F., 1888, Neue Methode zur Bestimmung der Aberrations-Constante nebst Untersuchungen über die Veränderlichkeit der Polhöhe, Beobachtungs-Ergebnisse der Königlichen Sternwarte zu Berlin, 3, 1-59.

McCarthy, D.D., and B.J. Luzum, 1996, Path of the mean rotational pole from 1899 to 1994, Geophys. J. Int., 125, 623-629.

Vicente, R. O., and C.R. Wilson, 1997, On the variability of the Chandler frequency, Journ. Geophys. Res., 102, 20439-20445.

Vondrák, J., 1999, Earth rotation parameters 1899.7-1992.0 after reanalysis within the HIPPARCOS frame, Surveys in Geophysics, 20, 169-195.

Vondrák, J., 2000, Survey of Observational Techniques and HIPPARCOS Reanalysis, this issue. 


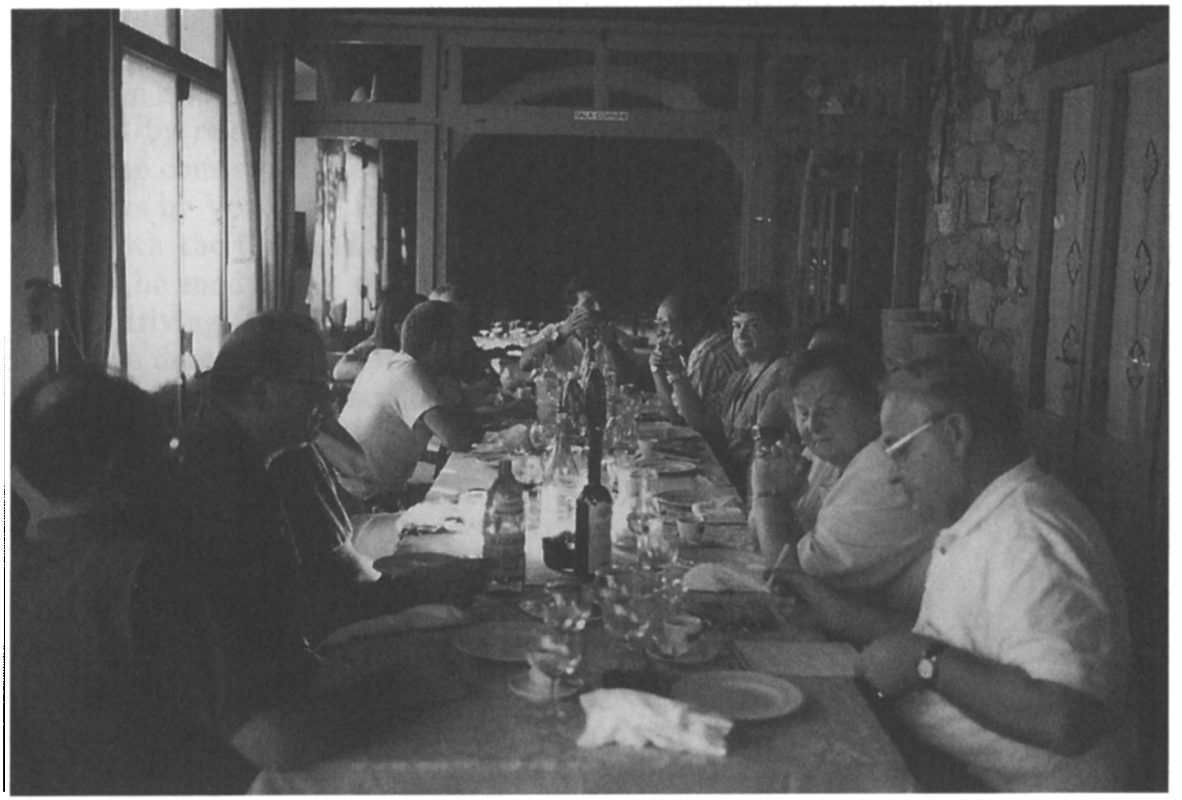

Excursion to Barumini megalithic village 